\title{
Can New Ultrasound Signs Help in Identifying Follicular Variant of Papillary Carcinoma of Thyroid? - A Pilot Study
}

Authors

Affiliations
C. Anuradha ${ }^{1}$, M. T. Manipadam ${ }^{2}$, H. S. Asha $^{3}$, N. Dukhabandhu ${ }^{3}$, D. Abraham ${ }^{4}$, M. J. Paul

${ }^{1}$ Radiology, Christian Medical College, Vellore, India

${ }^{2}$ Pathology, Christian Medical College, Vellore, India

${ }^{3}$ Endocrinology, Christian Medical College, Vellore, India

${ }^{4}$ Endocrine Surgery, Christian Medical College, Vellore, India
Key words

head/neck

thyroid

ultrasound received 21.05.2015

accepted $\quad 19.02 .2016$

Bibliography

DOI http://dx.doi.org/

10.1055/s-0036-1582304

Published online: 2016

Ultrasound International Open

2016; 2: E47-E53

(c) Georg Thieme Verlag KG

Stuttgart · New York

ISSN 2199-7152

\section{Correspondence}

Dr. Chandramohan Anuradha, DMRD, MD, DNB, FRCR

Department of Radiology Christian Medical College, Vellore

Radiology

Vellore

India 632004

Tel.:+ 91/41/62283 012

anuradhachandramohan@

gmail.com

\section{Abstract}

Aim: To describe two new ultrasound signs for thyroid nodules - "nodule in nodule" and "hypoechoic internal septae" and assess their usefulness in differentiating follicular variant of papillary thyroid carcinoma (FVPTC) from benign thyroid nodules (BTN).

Methodology: Ultrasound findings of 210 patients with histopathologically proven FVPTC (68 nodules, $\mathrm{M}: \mathrm{F}=13: 47$ with mean age of $39.5 \pm$ 11.9 years) and BTN (165 nodules, (M:F=41:109 with mean age of $44 \pm 11.3$ years) were retrospectively reviewed from PACS by a single radiologist blinded to the final diagnosis. Logistic regression analysis was performed to identify the best predictors of FVPTC and their diagnostic performance was assessed.

Results: The "nodule in nodule" sign was seen in $80.9 \%$ of FVPTC and only $12.1 \%$ of BTN. The "hypoechoic internal septae" sign was seen

\section{Introduction}

\section{$\nabla$}

Follicular variant of papillary carcinoma (FVPTC) is the second most common variant of papillary thyroid cancer (PTC) and constitutes $9-22.5 \%$ of all PTCs and it is a clinically distinct entity $[1,2]$. Histologically, FVPTCs are similar to PTC in that they show clear nuclei, nuclear grooves and pseudoinclusions like PTC, but they have follicular cell architecture [3]. Unlike papillary carcinoma of the thyroid, FVPTC has a relatively benign ultrasound appearance and is often missed by radiologists [1,3-7]. FVPTCs were previously described as hypoechoic or isoechoic, oval, well-defined lesions with halo and no microcalcifications $[1,7]$. In our practice, we have often noticed certain other ultrasound findings not described so far in nodules which turned out to be FVPTC. However, we do not know how commonly these are seen and whether they can be in $44.1 \%$ of FVPTC and $17 \%$ of BTN. Younger patients, heterogeneous echotexture, nodule in nodule sign, thick incomplete non-uniform halo and presence of significant nodes were the best predictors of FVPTC $(p<0.05)$. The sensitivity, specificity, positive predictive value, negative predictive value, accuracy and relative risk for FVPTC given as odds ratio $(95 \% \mathrm{CI})$ for heterogeneous echotexture and nodule in nodule sign which were the best 2 predictors of FVPTC were $91.2 \%, 81.8 \%, 67.3 \%, 95.7 \%, 84.5 \%, 46.5$ (18.5-117.4) and $80.9 \%, 87.7 \%, 74.3 \%, 91.2 \%$, $86.2 \%, 32.5$ (15.04-70.2), respectively. There was improvement in the specificity (91.5\%) and accuracy $(86.6 \%)$ when a combined criterion of heterogeneous echotexture and nodule in nodule sign was applied to predict FVPTC.

Conclusion: The "nodule in nodule" sign is common in FVPTC and when combined with heterogeneous echotexture can differentiate FVPTC and BTN with high specificity.

used to differentiate FVPTC from other benign thyroid nodules (BTN). In this study, we have aimed to assess the usefulness of two newly described ultrasound signs, "nodule in nodule" sign and "hypoechoic internal septae", to differentiate follicular variant of papillary carcinoma (FVPTC) from benign thyroid nodules (BTN) and also identify the best ultrasound findings for differentiating the two.

\section{Materials and Methods}

This is an institutional review board (IRB) approved retrospective study conducted in the department of radiology of a tertiary care teaching hospital with 2800 beds in South India. Informed consent was waived by IRB. The endocrine surgery database was searched for patients who received surgical histopathological diagno- 
sis of FVPTC or benign thyroid nodule between Jan 2011 and Jan 2013. A total of 60 patients received a diagnosis of FVPTC and a total of 338 patients received a histopathological diagnosis of BTN during the study period. Patients were included in the study only if they underwent preoperative ultrasound of thyroid gland in our institution. All 60 patients (with 68 nodules in 13 males and 47 females with mean age of $39.5 \pm 11.9$ years) who received a diagnosis of FVPTC and only alternative consecutive patients who received a diagnosis of BTN which was a total of 150 patients (with 165 nodules in 41 males and 109 females with mean age of $44 \pm 11.3$ years) with BTN were included in the study. Among patients with FVPTC, the majority $(81.7 \%)$ had encapsulated variety of FVPTC ( 49 patients with 54 nodules) and the rest (11 patients with 14 nodules) had a diffuse or invasive form of FVPTC. 5 patients with encapsulated FVPTC had capsular breach, 1 patient had lymphovascular invasion and 7 had metastatic neck nodes. Among the patients with a diffuse form of FVPTC, 6 patients had capsular breach, 2 patients had lymphovascular invasion, 1 had extrathyroidal extension and 5 had metastatic neck nodes on histopathology. 2 patients with the diffuse form of FVPTC had bone metastases at presentation. The histopathological diagnosis of various BTNs was: 97 (58.8\%) cases of nodular hyperplasia; 19 (11.5\%) hurthle cell adenomas, $3(1.8 \%)$ cases of Hashimoto's thyroiditis; 23 (13.9\%) follicular adenomas and $23(13.9 \%)$ cases of adenomatous hyperplasia.

Ultrasound of the thyroid was performed with a $7-12 \mathrm{MHz}$ highfrequency ultrasound probe. $\odot$ Table 1 gives the list of ultrasound machines that were used at our institution to perform thyroid ultrasound. Ultrasound images of these patients were reviewed from picture archiving and communication system (PACS) by a single consultant radiologist with 7 years of radiology experience. The radiologist was blind to the final diagnosis. Ultrasound findings of each nodule were described and documented. The ultrasound features that were assessed were: nodule composition (solid, cystic, mixed), echogenicity (hyperechoic, isoechoic, hypoechoic, markedly hypoechoic), echotexture (homogeneous, heterogeneous), margins (well-defined with or without halo sign, microlobulated, ill-defined, irregular), presence of calcification (microcalcification, macrocalcification) and shape of the nodule (taller than wide or wider than tall).

Nodules with $>75 \%$ solid component were labeled as solid. Cystic nodules had no solid components, and mixed nodules had both solid and cystic areas with solid component constitution $<75 \%$ of the size of the lesion. For mixed lesions echogenicity, margin, shape and presence of calcification were assessed for the solid component. Irrespective of the nodule composition, the presence of cystic change in the nodules was separately documented. Echogenicity was described in comparison with the

Table 1 Ultrasound equipment used for thyroid ultrasound at our institution.

\begin{tabular}{|c|c|c|}
\hline Model, manufacturer & $\begin{array}{l}\text { Frequency range of high- } \\
\text { frequency probe }(\mathrm{MHz})\end{array}$ & $\begin{array}{l}\text { Color } \\
\text { Doppler }\end{array}$ \\
\hline ACUSON Antares ${ }^{\mathrm{TM}}$, Siemens & $5-13,3-7$ & Yes \\
\hline ACUSON S2000M, Siemens & $5-14,6-9$ & Yes \\
\hline XARIO-ISTYLE, Toshiba & $7-14$ & Yes \\
\hline Xario XG, Toshiba & $7-14$ & Yes \\
\hline HD 11, Philips & $3-12$ & Yes \\
\hline LOGIQ 5 EXPERT, GE Health Care & $5-12$ & Yes \\
\hline LOGIQ P6 BT, GE Health Care & $7-12$ & Yes \\
\hline LOGIQ 500 PRO, GE Health Care & $7-12$ & Yes \\
\hline
\end{tabular}

thyroid gland and strap muscles. A lesion was considered hyperechoic if the echogenicity was more than that of the thyroid gland; isoechoic if the echogenicity was equal to thyroid gland; hypoechoic if the echogenicity was equal to strap muscle and markedly hypoechoic if the echogenicity was lower than the strap muscle. The echotexture was documented as homogeneous if the nodule had uniform echogenicity and heterogeneous if the echogenicity was variable ( $\boldsymbol{\bullet}$ Fig. 1 ). A hypoechoic smooth thin rim around the nodule was considered a positive halo sign. A halo sign was documented in detail and described if the halo was continuous or disrupted, uniform or non-uniform, thin $(<1 \mathrm{~mm})$ or thick $(>/=1 \mathrm{~mm})$ in nature ( $\bullet$ Fig. $\mathbf{1})$. Short cycle undulations of $>3$ along the margin were considered a microlobulated margin. A spiculated margin was considered irregular while fuzzy margins were considered ill-defined. A calcification measuring less than $1 \mathrm{~mm}$ was defined as a microcalcification and a calcification of more than $1 \mathrm{~mm}$ was labeled as a macrocalcification. The shape was described as taller than wide if the anterio-posterior dimension was equal to or greater than the transverse dimension and a nodule was described as wider than tall if the transverse dimension of the nodule was greater than the anterio-posterior dimension. Color Doppler findings were documented were available.

Nodules were then assessed for 2 novel signs - the "nodule in nodule" sign and the "hypoechoic internal septae" sign. The "nodule in nodule" sign ( $\boldsymbol{\bullet}$ Fig. 2 ) was considered positive if there were discrete hypoechoic areas within an isoechoic nodule. The "hypoechoic internal septa" sign ( $\bullet$ Fig. 3) was considered positive if the nodule had branching hypoechogenic septae within an isoechoic nodule.

\section{Statistical analysis}

IBM SPSS Analytics 16.0 software (Chicago, Ill., USA) was used for statistical analysis. Continuous variables were assessed using the Wisconsin rank sum test and categorical variables were assessed using the Chi square test. Binary logistic regression analysis was performed to identify the best ultrasound features which differentiate FVPTC from benign thyroid nodules and their diagnostic performance was assessed using ROC curve analysis.

\section{Results \\ $\nabla$}

- Table 2 summarizes the clinical and imaging features of FVPTC and BTN. The mean age of the patients with FVPTC (39.5 years) was significantly younger compared to BTN (44 years), $\mathrm{p}<0.001$. However, there was no difference in the sex distribution and the size of the nodules. 52 patients with FVPTC and 136 patients with BTN underwent FNAC. Our positive hit rate for FNAC was $65.4 \%$ and $79.4 \%$ for FVPTC and BTN, respectively. Among patients with FVPTC, 7.7\% $(n=4)$ had malignant cytology, 5.8\% $(n=3)$ had cytology which was suspicious for malignancy, $26.1 \%$ $(n=14)$ had atypia of undetermined significance or follicular lesions and $25 \%(n=13)$ had benign cytology. Among the patients with BTN, 50\% ( $n=68)$ had benign cytology, 28.6\% $(n=39)$ had atypia of undetermined significance or follicular lesions, only 1 patient $(0.7 \%)$ had cytology suspicious for malignancy and none had malignant cytology.

A large majority of FVPTCs were solid (86.5\%), isoechoic (80.9\%), well-defined $(69.1 \%)$, oval in shape $(61.8 \%)$, showed peripheral vascularity ( $86.3 \%$ ) and had no calcifications $(70.4 \%)$. The major- 

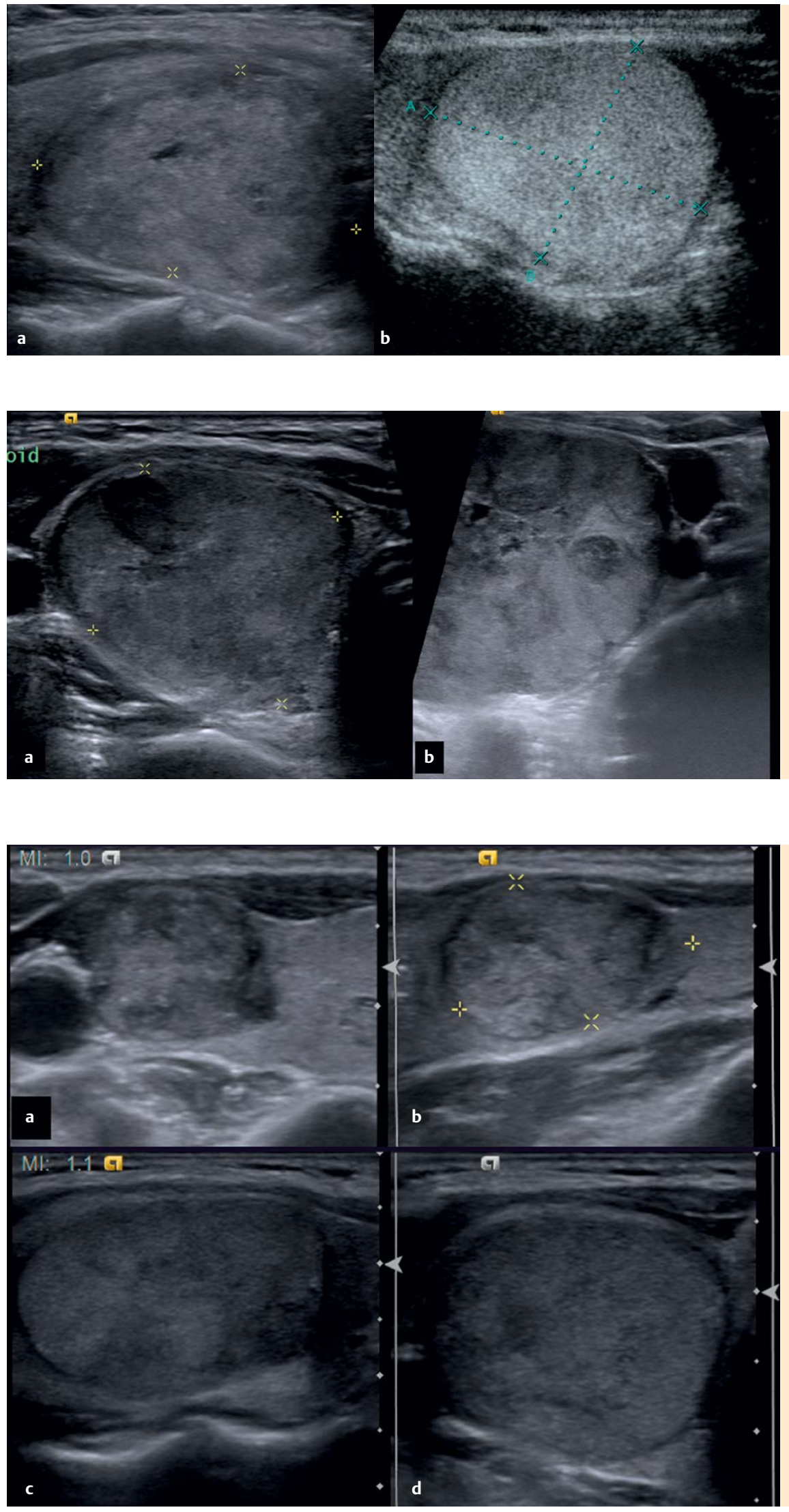

Fig. 1 a, b Ultrasound images of patients with encapsulated FVPTC and BTN demonstrating their echotexture and characteristics of halo sign on ultrasound a Ultrasound of thyroid of 36-year-old female patient with histopathological diagnosis of FVPTC shows a well defined isoechoic, oval thyroid nodule with heterogeneous echotexture and thick non uniform halo. $\mathbf{b}$ Ultrasound of thyroid of 42-year-old female patient with benign follicular adenoma shows a well defined, isoechoic, oval thyroid nodule with homogeneous echotexture and thin uniform halo.

Fig. 2 a, b Ultrasound images of thyroid nodules in 2 different patients with FVPTC showing positive nodule in nodule sign seen as ill-defined hypoechoic focus within a predominantly isoechoic well defined nodule.
Fig. 3 a-d Ultrasound images of thyroid nodule in 2 patients ( $\mathbf{a}, \mathbf{b}$ - patient 1 and $\mathbf{c}, \mathbf{d}$ - patient 2) with histopathological diagnosis of FVPTC showing positive hypoechoic internal septae sign which is seen as ill-defined hypoechoic branching echoes within a well defined isoechoic nodule. ity of FVPTCs (70.6\%) were reported as either probably benign $(41.2 \%)$ or a nodule with low suspicion for malignancy $(29.4 \%)$. Similarly, the majority of BTNs were solid (73.3\%), isoechoic (86.1\%), well-defined (98.8\%), oval in shape $(92.7 \%)$, showed peripheral vascularity (90\%) and had no calcification (90.3\%).
Most BTNs (83.7\%) were reported as either benign (19.5\%) or probably benign (64.2\%).

The majority $(41 / 54=75.9 \%)$ of encapsulated variety of FVPTC had no malignant features and were reported as benign. On the other hand 11 out of 14 nodules ( $78.5 \%$ ) which received histopa- 


\begin{tabular}{|c|c|c|c|}
\hline & FVPTC $(n=68)$ & BTN $(n=165)$ & P-value \\
\hline Age & $39.5 \pm 11.9$ years & $44 \pm 11.3$ years & 0.006 \\
\hline Sex & $M: F=14: 54$ & $M: F=43: 122$ & 0.377 \\
\hline Size of nodule & $3.8 \pm 1.76 \mathrm{~cm}$ & $3.5 \pm 1.44 \mathrm{~cm}$ & 0.141 \\
\hline Composition & & & Chi square $=9.8$ \\
\hline Cystic & 0 & $21(12.7 \%)$ & $p=0.007$ \\
\hline Solid & $59(86.8 \%)$ & $121(73.3 \%)$ & \\
\hline Mixed & $9(13.2 \%)$ & $23(13.9 \%)$ & \\
\hline Echogenicity & & & Chi square $=14.5$ \\
\hline Hyperechoic & - & $7(4.2 \%)$ & $p=0.002$ \\
\hline Isoechoic & $55(80.9 \%)$ & $142(86.1 \%)$ & \\
\hline Hypoechoic & $7(10.3 \%)$ & $11(6.7 \%)$ & \\
\hline Markedly hypoechoic & $6(8.8 \%)$ & $1(0.6 \%)$ & \\
\hline Margins & & & Chi square $=48.1$ \\
\hline Well defined & 47 (69.1\%) & 163 (98.8\%) & $p<0.001$ \\
\hline III-defined & $17(25 \%)$ & $2(1.2 \%)$ & \\
\hline Irregular & $4(5.9 \%)$ & - & \\
\hline Calcification & & & Chi square $=29.4$ \\
\hline Microcalcification & $10(14.7 \%)$ & - & $p<0.001$ \\
\hline Macrocalcification & $9(13.2 \%)$ & $16(9.7 \%)$ & \\
\hline Micro- and macrocalcification & $1(1.5 \%)$ & - & \\
\hline No calcification & $48(70.6 \%)$ & $149(90.3 \%)$ & \\
\hline Shape & & & Chi square $=33.8$ \\
\hline Taller than wide & $26(38.2 \%)$ & $12(7.3 \%)$ & $p<0.001$ \\
\hline Wider than tall & $42(61.8 \%)$ & $153(92.7 \%)$ & \\
\hline Vascularity & & & 0.543 \\
\hline Central & $7(13.7 \%)$ & $6(10 \%)$ & \\
\hline Peripheral & $44(86.3 \%)$ & $54(90 \%)$ & \\
\hline Lymph nodes & $12(17.6 \%)$ & $2(1.2 \%)$ & $\begin{array}{l}\text { Chi square }=71.4 \\
p<0.001\end{array}$ \\
\hline Echotexture & & & Chi square $=108.1$ \\
\hline Homogeneous & $6(8.8 \%)$ & 83 (50.3\%) & $<0.001$ \\
\hline Heterogeneous & $62(91.2 \%)$ & $30(18.2 \%)$ & \\
\hline Honeycomb-like & - & $41(28.4 \%)$ & \\
\hline Anechoic & - & $11(6.7 \%)$ & \\
\hline Nodule in nodule sign & $55(80.9 \%)$ & $20(12.1 \%)$ & $\begin{array}{l}\text { Chi square }=106.9 \\
p<0.001\end{array}$ \\
\hline Hypoechoic internal septa sign & $30(44.1 \%)$ & $28(17 \%)$ & $\begin{array}{l}\text { Chi square }=18.9 \\
p<0.001\end{array}$ \\
\hline Presence of cystic change & $32(47.1 \%)$ & $116(70.3 \%)$ & $\begin{array}{l}\text { Chi square }=11.23 \\
p=0.001\end{array}$ \\
\hline Halo sign & $51(75 \%)$ & $150(90.9 \%)$ & $\begin{array}{l}\text { Chi square }=10.3 \\
p=0.001\end{array}$ \\
\hline Thick, non-uniform, incomplete halo & $54(79.4 \%)$ & $25(15.1 \%)$ & $\begin{array}{l}\text { Chi square }=88.7 \\
P<0.001\end{array}$ \\
\hline
\end{tabular}

Table 2 Comparison of age, sex and ultrasound features between FVPTC and BTN.
Table 3 Results of binary logistic regression analysis.

\begin{tabular}{|lccr|} 
Variables & B-value & Wald's statistics & P-value \\
\hline Age & -0.016 & 5.803 & 0.016 \\
\hline Heterogeneous echotexture & -2.26 & 7.824 & 0.005 \\
\hline $\begin{array}{l}\text { Nodule in nodule sign } \\
\text { Thick incomplete }\end{array}$ & 16.97 & 14.64 & 0.001 \\
$\begin{array}{l}\text { non-uniform halo } \\
\text { Cystic change in the nodule }\end{array}$ & -1.89 & 8.935 & 0.003 \\
\hline Hypoechoic internal septae & 0.243 & 0.151 & 0.698 \\
\hline Calcification & 20.19 & 1.158 & 0.282 \\
\hline Vascularity & -1.893 & 0.000 & 0.999 \\
\hline Lymph nodes & -2.46 & 1.228 & 0.268 \\
\hline Cox and & & & 0.000
\end{tabular}

Cox and snell $R^{2}=0.567$

Hosmer and Lemeshow goodness of fit test: chi square $=17.565, \mathrm{df}=8, \mathrm{p}=0.025$

thology diagnosis of invasive variety of FVPTC had one or more malignant features on ultrasound. This difference between the encapsulated and invasive variety of FVPTC was statistically sig- nificant (chi square=39, p<0.001). 5 FVPTCs were within a benign thyroid nodule which was either nodular hyperplasia $(n=4)$ or Hurthle cell adenoma $(n=1) .3$ of these were microscopic foci of FVPTC in nodular hyperplasia.

Heterogeneous echotexture (91.2\% of FVPTC vs. $18.2 \%$ of BTN), nodule in nodule sign ( $80.1 \%$ of FVPTC vs. $12.1 \%$ of BTN), hypoechoic internal septa sign (44.1\% of FVPTC vs. $17 \%$ of BTN) and thick non-uniform incomplete halo (79.4\% of FVPTC vs. $15.1 \%$ of BTN) were findings that were significantly more common in FVPTC ( $\mathrm{p}<0.001)$. Honeycomb appearance (none of FVPTC vs. $28.4 \%$ of BTN), cystic change (47.1\% of FVPTC vs. $70.3 \%$ of BTN) and halo sign ( $75 \%$ of FVPTC vs. $90.9 \%$ of BTN) were significantly more common in BTN $(\mathrm{p}<0.001)$.

- Table 3 shows the results of binary logistic regression analysis. Of all the features, age, heterogeneous echotexture, positive nodule in nodule sign, thick non-uniform incomplete halo, and presence of significant nodes were the best predictors of FVPTC $(p<0.005)$. Echogenicity of the nodule, cystic change, hypoechoic internal septa sign, vascularity and calcification were not 
Table 4 Diagnostic performance of top predictors of FVPTC.

Echotexture

Nodule in nodule sign

Absent or thick, incomplete and non-uniform halo

Hypoechoic septa sign

Lymph nodes

Combined criteria 1 (heterogeneous echotexture + positive

nodule in nodule sign)

Combined criteria 2 (heterogeneous echotexture + positive nodule in

nodule sign + absent or thick, incomplete and non-uniform halo)

\begin{tabular}{lcccccc}
$\begin{array}{l}\text { Odds ratio for } \\
\text { FVPTC }(\mathbf{9 5} \% \mathbf{C l})\end{array}$ & Sensitivity & Specificity & PPV & NPV & Accuracy & ROC AUC \\
\hline $46.5(18.5-117.4)$ & 91.2 & 81.8 & 67.3 & 95.7 & 84.5 & 0.902 \\
\hline $32.5(15.04-70.2)$ & 80.9 & 87.7 & 74.3 & 91.2 & 86.2 & 0.795 \\
\hline $21.6(10.4-44.6)$ & 78.5 & 84.8 & 67.1 & 90.9 & 83.2 & 0.686 \\
\hline $3.86(2.06-7.03)$ & 44.12 & 83 & 51.7 & 78.2 & 71.6 & 0.648 \\
$17.4(3.79-80.4)$ & 17.5 & 98.7 & 85.7 & 74.7 & 73.1 & 0.562 \\
\hline $32.3(14.9-70.2)$ & 75 & 91.5 & 78.4 & 89.9 & 86.6 & 0.833 \\
& & & & & & \\
\hline $42.8(16.5-110.7)$ & 61.7 & 96.3 & 87.5 & 85.9 & 96.2 & 0.791 \\
& & & & & & \\
\hline
\end{tabular}

useful. $\odot$ Table 4 shows the diagnostic performance of the significant predictors of FVPTC.

\section{Discussion}

\section{$\nabla$}

FVPTC constitutes the second most common variant of papillary carcinoma of thyroid (PTC) next only to the classic variant of PTC and was first described by Crile and Hazard in 1953 [8]. Since then, the diagnosis of FVPTC has always been a diagnostic challenge for clinicians, pathologists and radiologists [9]. Clinically, FVPTC presents mostly as solitary nodules incidentally detected in patients around 40 years of age with male to female ratio of 1:6. Lymph node metastases precede lung metastases in FVPTC and the risk of regional nodal metastases is lower than PTC. Compared to patients with conventional PTC, patients with FVPTC have a better tumor risk group profile. However, the overall long-term survival among both types of PTC is similar $[10,11]$. Diagnosis of FVPTC is not possible on FNAC and always needs surgical excision for definitive diagnosis [12]. In our series, very few patients with FVPTC had malignant cytology or cytology suspicious for malignancy. Nearly half of patients with FVPTC had either benign cytology or indeterminate cytology with atypical cells. FVPTCs have a unique histological appearance where the cells have a follicular architecture but the nuclei features are that of PTC. Although pathologists agree widely on the diagnostic criteria for FVPTC, concordance even among experienced pathologists is known to be variable and as low as $40 \%$ [13]. Difficulty in differentiating between follicular adenomas and follicular carcinomas which are a close pathological differential diagnosis for FVPTC is the probable cause for the low concordance among pathologists [4].

FVPTCs are more commonly well encapsulated tumors with or without capsular invasion and less commonly they are diffusely infiltrative, as also seen in our series. Often, a histopathological diagnosis of FVPTC comes as a surprise to the sinologist. The majority (76\%) of encapsulated variety of FVPTC had no malignant features on ultrasound and were reported as benign in our series. On the other hand, $79 \%$ of nodules which received histopathology diagnosis of invasive variety of FVPTC had one or more malignant features on ultrasound. FVPTCs have been described as well-defined, oval, isoechoic or hypoechoic nodules on ultrasound and less than $50 \%$ showed one or more malignant ultrasound features $[1,4]$. In our practice we have often noted a few previously undescribed ultrasound findings, i.e., the "nodule in nodule" sign and "hypoechoic internal septae" sign, in thyroid nodules which turned out to be FVPTC. This study was done to see the usefulness of these signs in differentiating FVPTC from BTN. We also went on to identify the best ultrasound predictors of FVPTC.

The first sign - the "nodule in nodule" sign - represents discrete, hypoechoic or markedly hypoechoic areas within an isoechoic, well-defined thyroid nodule. This sign was very commonly $(80 \%)$ seen in FVPTC and was uncommon in BTN (12\%). A similar nodule in nodule appearance was also seen in the cut section of a thyroidectomy specimen of histopathologically proven FVPTC of thyroid ( $\bullet$ Fig. 4). We know that highly cellular tumors, both benign and malignant, appear hypoechoic on ultrasound. Histopathologically, FVPTC has PTC like cells arranged in follicles and these follicles have variable amounts of colloid in the center of the follicles. We think that the histopathological reason for the nodule in nodule sign is tightly packed follicles with absent central colloid making that region more cellular compared to other areas with colloid in the follicles ( $\bullet$ Fig. 4). However, we need focused radio-pathological correlation studies to prove this. Another possible pathological reason for this sign may be FVPTC arising within a BTN. However, only 5 FVPTCs were within benign thyroid nodules in our series and thus may not explain the commonality of this sign.

The second sign - the "hypoechoic internal septae" sign - represents an ill-defined, hypoechoic branching septae like echoes within a well-defined isoechoic nodule. This sign was seen in $44 \%$ of FVPTCs and $17 \%$ of BTNs and its diagnostic performance was just fair (AUC $=0.648$ ). The probable reason for this sign may be tumor, both FVPTC and BTN occurring in the background of diffuse thyroid disease which has fibrous septations within the gland. Studying for associated changes of diffuse thyroid disease, however, was beyond the scope of this study.

Of all the features we studied, regression analysis showed that the presence of heterogeneous echotexture, nodule in nodule sign, thick non-uniform incomplete halo and significant nodes were the best ultrasound predictors of FVPTC $(p<0.005)$. Other findings like the presence of cystic change, calcification, and hypoechoic internal septae were not useful.

Heterogeneous echotexture was very commonly (91\%) seen in FVPTC and less commonly (18\%) in BTN. Heterogeneity, either heterogeneous echotexture or heterogeneous contrast enhancement in CT or MRI has been noted as a useful sign to differentiate malignant from benign lesions. For example, this is most useful in differentiating fibrolamellar hepatocellular carcinoma from focal nodular hyperplasia. Similarly, we found heterogeneous echotexture more commonly in FVPTC. The heterogeneous echotexture is probably due to the non-uniform arrangement of follicles, non-uniform size of the nucleus and heterogeneous cell 


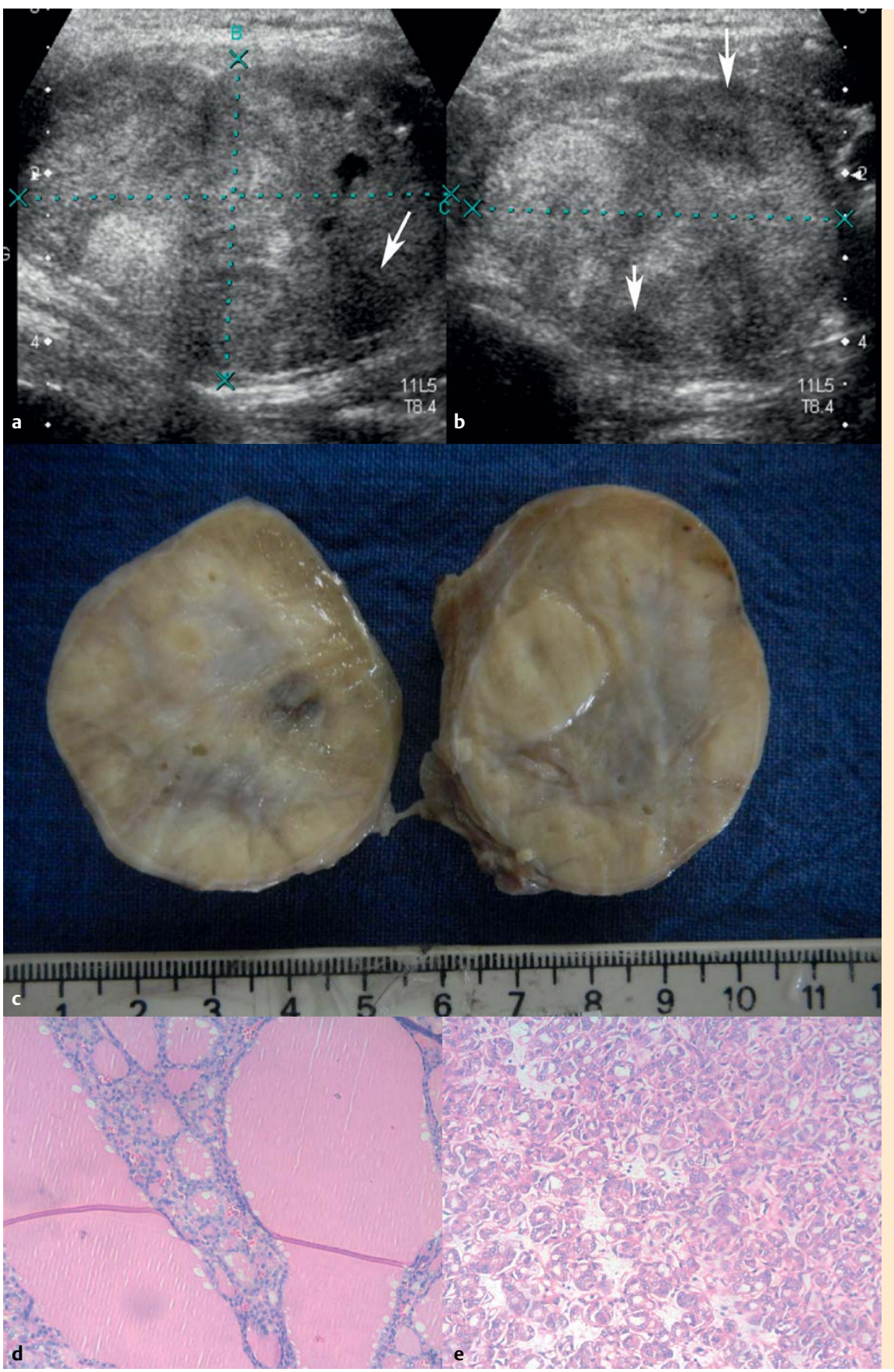

Fig. 4 a, b A 43-year-old female patient with encapsulated FVPTC. $\mathbf{a}, \mathbf{b}$ Ultrasound of the thyroid shows well defined, oval, isoechoic nodule with heterogeneous echotexture and positive nodule in nodule sign. c Cut section of total thyroidectomy specimen showing nodule in nodule appearance. $\mathbf{d}$ Photomicrograph of hematoxylin and eosin stained slides, $\times 200$ magnification of FVPTC showing follicles rich in colloid corresponding to isoechoic region of the nodule on ultrasound. e Photomicrograph of hematoxylin and eosin stained slides, $\times 200$ magnification of FVPTC showing cellular region of the tumor where follicles lack colloid corresponding to the hypoechoic area within the isoechoic nodule (nodule in nodule) on ultrasound.

density throughout the FVPTC which is malignant. On the other hand, BTNs are homogeneous due to uniformity in the morphology of the cells and their arrangement. Vito Cantisani et al. in their studies have also demonstrated similar usefulness of mixed or heterogeneous echotexture, irregular margins and strain ratio $>2.05$ in differentiating malignant and benign thyroid nodules with indeterminate cytology. They also showed the limitations of elastography in identifying follicular carcinomas since they are relatively soft [14-16]. However, their study included nodules with a wide range of histology including FVPTC.

Although the halo sign was seen in $75 \%$ of FVPTCs and more than $90 \%$ of BTNs, the morphology of the halo sign was different between the 2 . Nearly $80 \%$ of FVPTCs had a thick, non-uniform halo while the halo was uniform in $85 \%$ of BTNs. The non-uniform nature of the halo sign seen in FVPTC could be related to the presence of microscopic capsular invasion. The presence of metastatic neck nodes is a definite sign of malignancy, however was seen in only $18 \%$ of patients with FVPTC.

The diagnostic performance of heterogeneous echotexture was excellent (AUC of 0.902) followed by the nodule in nodule sign whose performance was good (AUC of 0.795). While the sensitivity of heterogeneous echotexture (91\%) was higher than the nodule in nodule sign ( $81 \%)$, the specificity for the nodule in nodule sign $(88 \%)$ was higher than heterogeneous echotexture (82\%). There was marginal improvement of diagnostic accuracy (86.6\%) and excellent diagnostic performance (AUC=0.833) when the combined criterion of heterogeneous echotexture and nodule in nodule sign was applied. However, adding the halo sign to the above combined criterion did not improve diagnostic accuracy (86.2\%) or performance. 
There are few limitations in this study. The retrospective nature of the study is a limitation. We had to go by images archived on PACS and ultrasound features may not have been adequately documented. Doppler findings were not documented in all patients and the value of color Doppler findings could not be fully brought out in this study. We did not match the nodules for size or patient characteristics and this is a limitation. The interand intra-observer variability among pathologists for the diagnosis of FVPTC even among experts is well known. This may have led to misclassification of a few patients.

In conclusion, heterogeneous echotexture, positive nodule in nodule sign, thick non-uniform incomplete halo and presence of significant lymph nodes were useful ultrasound findings to differentiate FVPTC and BTN. Heterogeneous echotexture and nodule in nodule sign or a combination of these 2 signs was the most useful in making an ultrasound diagnosis of FVPTC. The presence of these signs in a well-defined, benign appearing thyroid nodule should make thyroid sonologists consider FVPTC. This may prompt pathology colleagues to perform genetic tests to look for RAF/ BRAF mutations which will help them better characterize these lesions and may similarly prompt surgical colleagues to be more aggressive in the management of these nodules. Also, further prospective radio-pathological correlation studies are needed to validate the findings of this study.

\section{Acknowledgements}

$\nabla$

We acknowledge the statistical assistance provided by the department of biostatistics of Christian Medical College, Vellore, India.

\section{References}

1 Yoon JH, Kim E-K, Hong SW et al. Sonographic features of the follicular variant of papillary thyroid carcinoma. J Ultrasound Med Off J Am Inst Ultrasound Med 2008; 27: 1431-1437

2 Komatsu M, Hanamura N, Tsuchiya S et al. Preoperative diagnosis of the follicular variant of papillary carcinoma of the thyroid: discrepancy between image and cytologic diagnoses. Radiat Med 1994; 12 : 293-299
3 Ozdemir D, Ersoy $R$, Cuhaci $N$ et al. Classical and follicular variant papillary thyroid carcinoma: comparison of clinical, ultrasonographical, cytological, and histopathological features in 444 patients. Endocr Pathol 2011; 22: 58-65

$4 \mathrm{Kim}$ DS, Kim J, Na DG et al. Sonographic features of follicular variant papillary thyroid carcinomas in comparison with conventional papillary thyroid carcinomas. J Ultrasound Med Off J Am Inst Ultrasound Med 2009; 28: 1685-1692

5 Lee S, Han B-K, Ko EY et al. The ultrasonography features of hyalinizing trabecular tumor of the thyroid are more consistent with its benign behavior than cytology or frozen section readings. Thyroid Off J Am Thyroid Assoc 2011; 21: 253-259

6 Rago T, Di Coscio G, Basolo F et al. Combined clinical, thyroid ultrasound and cytological features help to predict thyroid malignancy in follicular and Hupsilonrthle cell thyroid lesions: results from a series of 505 consecutive patients. Clin Endocrinol (Oxf) 2007; 66: 13-20

7 Yang GCH, Fried K, Yakoushina TV et al. Encapsulated follicular variant of papillary thyroid carcinoma: fine-needle aspiration with ultrasound and histologic correlation of 41 cases. Acta Cytol 2013; 57: 26-32

8 Crile G, Hazard JB. Relationship of the age of the patient to the natural history and prognosis of carcinoma of the thyroid. Ann Surg 1953; 138: $33-38$

9 Liu J, Singh B, Tallini G et al. Follicular variant of papillary thyroid carcinoma: a clinicopathologic study of a problematic entity. Cancer 2006; 107: 1255-1264

10 Lang BH-H, Lo C-Y, Chan W-F et al. Classical and follicular variant of papillary thyroid carcinoma: a comparative study on clinicopathologic features and long-term outcome. World J Surg 2006; 30: 752-758

11 Passler C, Prager G, Scheuba C et al. Follicular variant of papillary thyroid carcinoma: a long-term follow-up. Arch Surg Chic Ill 1960. 2003; 138: $1362-1366$

12 Martínez-Parra D, Campos Fernández J, Hierro-Guilmain CC et al. Follicular variant of papillary carcinoma of the thyroid: to what extent is fine-needle aspiration reliable? Diagn Cytopathol 1996; 15: 12-16

13 Lloyd RV, Erickson LA, Casey MB et al. Observer variation in the diagnosis of follicular variant of papillary thyroid carcinoma. Am J Surg Pathol 2004; 28: 1336-1340

14 Grazhdani H, Cantisani V, Lodise $P$ et al. Prospective evaluation of acoustic radiation force impulse technology in the differentiation of thyroid nodules: accuracy and interobserver variability assessment. J Ultrasound 2014; 17: 13-20

15 Cantisani V, Grazhdani H, Drakonaki E et al. Strain US Elastography for the Characterization of Thyroid Nodules: Advantages and Limitation. Int J Endocrinol 2015 2015: 908575

16 Cantisani V, Ulisse S, Guaitoli E et al. Q-elastography in the presurgical diagnosis of thyroid nodules with indeterminate cytology. PloS One 2012; 7: e50725 